\title{
ENERGY STABILITY OF BENARD-DARCY TWO-COMPONENT CONVECTION OF MAXWELL FLUID
}

\author{
H. MUTI and H. DEMIR* \\ Department of Mathematics, Art and Science Faculty \\ Ondokuz Mayis University \\ 55139 Atakum/Samsun-TURKEY \\ E-mail: hdemir@omu.edu.tr; hmuti@omu.edu.tr \\ P.G. SIDDHESHWAR \\ Bangalore University, Central College Campus \\ Bangalore 560 001, INDIA \\ E-mail: pgsmath@gmail.com
}

\begin{abstract}
Energy stability of a horizontal layer of a two-component Maxwell fluid in a porous medium heated and salted from below is studied under the Oberbeck-Boussinesq-Darcy approximation using the Lyapunov direct method. The effect of stress relaxation on the linear and non-linear critical stability parameters is clearly brought out with coincidence between the two when the solute concentration is dilute. Qualitatively, the result of porous and clear fluid cases is shown to be similar. In spite of lack of symmetry in the problem it is shown that non linear exponential stability can be handled.
\end{abstract}

Key words: energy stability analysis, Lyapunov direct method, B'enard-Darcy convection, viscoelastic, twocomponent Maxwell fluid, stress relaxation.

Mathematics Subject Classification (2000):76R10, 76E06, 76S05

\section{Introduction}

A free convective flow of a viscoelastic fluid through closely packed porous media is an important area rheometrically due to the fact that the order of the differential equation is smaller than that of loosely packed porous media. Oil recovery processes, mantle convection and other allied areas are typical situations wherein viscoelastic fluid convection can be important. Rayleigh-Bénard convection in viscoelastic liquids with two relaxation times (Jeffrey liquid) or one relaxation time (either Maxwell or Rivlin-Ericksen liquids) is thus important from the practical point of view. An analogous problem in Newtonian fluid-saturated porous media is now well investigated (see the monographs by Bejan (2004), Nield and Bejan (2006), Vafai (2000) and Alloui et al. (2010), and references therein). Some aspects of the problem involving a viscoelastic liquid were studied by Sharma and Sunil (1994), Haro et al. (1996), Srikrishna (2001), Siddheshwar and Srikrishna (2001)-(2003), Kim et al. (2003), Kumar and Singh (2006), Fu et al. (2007), Shivakumara and Sureshkumar (2008), Long et al. ((2009)-(2008)), Malshetty et al. (2009), Malashetty and Kulkarni (2009), Malashetty et al. (2009), Tan and Masuoka (2007), Wang and Tan (2008), and Awad et al. (2010). The main result that can be conjured up from these works focusing on the onset of convection is that Maxwell fluids are generally more unstable than Jeffreys liquids while Rivlin-Ericksen and Newtonian liquids are comparatively more stable. Further, in the case of a Jeffreys liquid occupying porous media it was found that for oscillatory convection to preferentially set in, the strain retardation time must be less than the stress relaxation time.

\footnotetext{
* To whom correspondence should be addressed
} 
There are fewer works on the nonlinear realm of Rayleigh-Bénard convection of two-component viscoelastic fluid convection. The present work addresses energy stability of the problem. Information on the coincidence or otherwise of the linear and nonlinear marginal stability curves in viscoelastic fluid convection through porous media provides good ideas to experimenters on situations conducive for rheometric measurements. The present paper aims to provide such useful information on the problem at hand.

\section{Basic equations}

Let $d>0, \Omega=I R^{2} \times(-d / 2, d / 2)$ and $0 x y z$ be a Cartesian frame of reference with unit vectors $i, j$, $k$ in the $x-, y-$, and $z-$ directions respectively. We assume that the Oberbeck-Boussinesq approximation is valid and that the flow in the porous medium is governed by Maxwell-Darcy's law. Thus, the basic equations for the problem at hand are

$$
\begin{aligned}
& \frac{\mu}{K} \boldsymbol{v}=\left(1+\lambda \frac{\partial}{\partial t}\right)\left(\nabla P-\rho_{f} g \boldsymbol{k}\right), \\
& \frac{1}{M} \frac{\partial T}{\partial t}+\boldsymbol{v} \nabla T=k \Delta T, \\
& \varepsilon \frac{\partial C}{\partial t}+\boldsymbol{v} \nabla C=k^{\prime} \Delta C, \\
& \nabla \boldsymbol{v}=\boldsymbol{0}, \\
& \rho_{f}=\rho_{0}\left(1-\alpha\left(T-T_{0}\right)+\alpha^{\prime}\left(C-C_{0}\right)\right)
\end{aligned}
$$

where $\rho_{0}$ is the density at the reference state, $\lambda$ is a stress relaxation constant, $v=(U, V, W)$ is the velocity, $P$ is the pressure, $T$ is the temperature and $C$ is the concentration, $M$ is the ratio of heat capacities as defined by Lombardo et al. (2001), and $\varepsilon$ is the normalized porosity defined by $\varepsilon=\varepsilon^{\prime} M$ where $\varepsilon^{\prime}$ is the porosity as in Lombardo et al. (2001). The quantities $\mu$ and $K$ denote the viscosity of the fluid and permeability of the porous medium, $g$ is the gravitation acceleration, $k$ and $k^{\prime}$ are the effective thermal diffusivity and solutal diffusivity of a Maxwell fluid saturated porous medium as defined in Lombardo et al. (2001), $\alpha$ and $\alpha^{\prime}$ are the coefficients for thermal and solutal expansion, and $T_{0}$ and $C_{0}$ are the reference temperature and concentration, respectively. Further $T_{1}=T_{0}+\Delta T, T_{2}=T_{0}-\Delta T, C_{1}=C_{0}+\Delta C C_{2}=C_{0}-\Delta C$.

The boundary conditions of the problem are

$$
W=0, \quad T=T_{0} \pm \Delta T, \quad C=C_{0} \pm \Delta C \quad \text { at } \quad z=\mp \frac{d}{2} .
$$

We now define non-dimensional quantities by

$$
\begin{array}{ll}
(\tilde{x}, \tilde{y}, \tilde{z}) \equiv \tilde{\boldsymbol{x}}=\frac{x}{d}, \quad \tilde{t}=\frac{k M}{d^{2}} t, \quad \tilde{\boldsymbol{v}}=\frac{d}{k} \boldsymbol{v}, \quad \tilde{\lambda}=\frac{d^{2}}{k M} \lambda, \\
\tilde{T}=\frac{T-T_{0}}{2 \Delta T}, \quad \tilde{C}=\frac{C-C_{0}}{2 \Delta C}, \quad \tilde{P}=\frac{K\left(P+\rho_{0} g z\right)}{\mu k} .
\end{array}
$$


If we omit all tildes for simplicity, the governing Eqs in (2.1) now take the following dimensionless form

$$
\begin{aligned}
& \boldsymbol{v}=\left(1+\lambda \frac{\partial}{\partial t}\right)(-\nabla P+(\mathfrak{R} T-\wp C) \boldsymbol{k}) \\
& \frac{\partial T}{\partial t}+\boldsymbol{v} \nabla T=\Delta T \\
& \frac{\partial C}{\partial t}+\boldsymbol{v} \nabla C=\frac{1}{\mathrm{Le}} \Delta C \\
& \nabla \boldsymbol{v}=\boldsymbol{0}
\end{aligned}
$$

where $\Re=(2 \alpha g \Delta T d K / v k)$ and $\wp=\left(2 \alpha^{\prime} g \Delta C d k / v k\right)$ are thermal and solutal Rayleigh numbers, respectively, and $v=\left(\mu / \rho_{0}\right)$ is the kinematic viscosity. In writing the first of the Eqs in (2.4) use has been made of the fifth equation of (2.1).

The boundary conditions (2.2) in dimensionless form are

$$
W=0, \quad T= \pm \frac{1}{2}, \quad C= \pm \frac{1}{2} \quad \text { at } \quad z=\mp \frac{1}{2} .
$$

The motionless state solution of Eq.(2.4), subject to Eq.(2.5) is

$$
\boldsymbol{v}=\mathbf{0}, \quad T=-z, \quad C=-z, \quad\left(1+\lambda \frac{\partial}{\partial}\right)(\nabla P+z(\Re-\wp) \boldsymbol{k})=0 .
$$

We now move on to study the stability of this motionless state by superposing finite amplitude perturbation on it that is given by $\left(\boldsymbol{u}, \vartheta, \gamma, p_{2}\right)$. This procedure yields the governing equation for perturbation in the form

$$
\begin{aligned}
& \boldsymbol{u}=-\left(1+\lambda \frac{\partial}{\partial t}\right)\left(\nabla p_{2}-(\mathfrak{R} \vartheta-\operatorname{Le} \wp \gamma) \boldsymbol{k}\right), \\
& \frac{\partial \vartheta}{\partial t}+\boldsymbol{u} \nabla \vartheta=w+\Delta \vartheta, \\
& \varepsilon \operatorname{Le} \frac{\partial \gamma}{\partial t}+\operatorname{Le} \boldsymbol{u} \nabla \gamma=w+\Delta \gamma, \\
& \nabla \boldsymbol{u}=\boldsymbol{0}
\end{aligned}
$$

where $\boldsymbol{u}=(u, v, w)$ and use has been made of the transformation $\gamma \rightarrow$ Le $\gamma$. The boundary conditions for solving Eq.(2.7) are

$$
w=0, \quad \vartheta=\gamma=0 \quad \text { at } \quad z=\mp \frac{1}{2} .
$$


We assume that the perturbations $\boldsymbol{u}, \vartheta, \gamma, p_{2}$ are periodic functions of $x$, and $y$ of periods $2 \pi / a_{x}$ and $2 \pi / a_{y}$, respectively, $\left(a_{x}>0, a_{y}>0\right)$. In this study, $\Omega$ represents the cell defined as $\Omega=\left[0,2 \pi / a_{x}\right] \times\left[0,2 \pi / a_{y}\right] \times[-1 / 2,1 / 2]$ and the wave number $a=\left(a_{x}^{2}+a_{y}^{2}\right)$ is as defined in Lombardo $e t$ al. (2001). Having developed the governing equation for the finite amplitude perturbations of the binary fluid system, we now make a global stability analysis of the same in what follows.

\section{Global non-linear stability analysis} variables

To set the stage for a global stability analysis, we introduce the following change of dependent

$$
\phi=\mathfrak{R} \vartheta-\widehat{\wp} \gamma, \quad \psi=\mathfrak{R} \vartheta-\operatorname{Le} \delta \oint \gamma
$$

where we have used the transformation $\widehat{\wp} \rightarrow \operatorname{Le} \oint$. With $\delta$ Le $\neq 1$, we obtain from Eq.(2.7) the following equations

$$
\begin{aligned}
& \boldsymbol{u}=-\left(1+\lambda \frac{\partial}{\partial t}\right)\left(\nabla p_{2}-\phi \boldsymbol{k}\right) \\
& \operatorname{Le}\left(\phi_{t}+\boldsymbol{u} \nabla \phi\right)=(\operatorname{Le} \Re-\wp) w+\frac{\operatorname{Le}^{2} \delta-1}{\operatorname{Le} \delta-1} \Delta \phi-\frac{\mathrm{Le}-1}{\operatorname{Le}^{2} \delta-1} \Delta \psi, \\
& \psi_{t}+\boldsymbol{u} \nabla \psi=(\mathfrak{R}-\varsigma \delta) w+\frac{\delta(\mathrm{Le}-1)}{\operatorname{Le} \delta-1} \Delta \phi+\frac{\delta-1}{\operatorname{Le} \delta-1 \Delta \psi}, \\
& \nabla \boldsymbol{u}=\boldsymbol{0} .
\end{aligned}
$$

We first eliminate $p_{2}$ from the first of Eq.(3.2) and arrive at an equation involving only $w$. Multiplying the resulting equation by $\kappa w$ and the second and third Eqs of (2.7) by $\phi$ and $\mu_{l} \psi$, where $\kappa>0$ and $\mu_{l}$ are coupling constants, and integrating over $\Omega$, we get

$$
\begin{aligned}
& \kappa\left(1+\lambda \frac{\partial}{\partial t}\right)(\phi, w)-\kappa \lambda\left(\phi, \frac{\partial w}{\partial t}\right)=\kappa\|w\|^{2}, \\
& \operatorname{Le} \frac{d}{d t} \frac{1}{2}\|\phi\|^{2}=(\operatorname{Le} \Re-\wp)(w, \phi)-\frac{\operatorname{Le}^{2} \delta-1}{\operatorname{Le} \delta-1}\|\nabla \phi\|^{2}+\frac{\operatorname{Le}-1}{\operatorname{Le}^{2} \delta-1}(\nabla \psi, \nabla \phi), \\
& \mu_{1} \frac{d}{d t} \frac{1}{2}\|\psi\|^{2}=\left(\mu_{1} \Re-\wp \delta\right)(w, \psi)-\mu_{1} \frac{\delta(\operatorname{Le}-1)}{\operatorname{Le} \delta-1}(\nabla \psi, \nabla \phi)-\mu_{1} \frac{\delta-1}{\operatorname{Le} \delta-1}\|\nabla \psi\|^{2} .
\end{aligned}
$$

Introducing the following notation for cumulative energy 


$$
E(t)=\frac{1}{2}\left[\operatorname{Le}\|\phi\|^{2}+\mu_{1}\|\psi\|^{2}\right]
$$

we may combine the last two Eqs in (3.3) to get

$$
\dot{E}(t)=\alpha_{l}(w, \phi)+\beta(w, \psi)-\left[\Gamma\|\nabla \phi\|^{2}+\xi\|\nabla \psi\|^{2}+\eta(\nabla \phi, \nabla \psi)\right]
$$

where an overdot denotes a time derivative and $\alpha_{1}, \beta, \xi, \eta$ and $\Gamma$ are given by

$$
\begin{aligned}
& \alpha_{1}=\operatorname{Le} \Re-\widehat{\wp}, \quad \beta=\mu_{1}(\Re-\wp \delta), \quad \xi=\mu_{1} \frac{\delta-1}{\operatorname{Le} \delta-1}, \\
& \eta=\frac{(\mathrm{Le}-1)\left(\mu_{1} \delta-1\right)}{\mathrm{Le} \delta-1}, \quad \Gamma=\frac{\mathrm{Le}^{2} \delta-1}{\operatorname{Le} \delta-1} .
\end{aligned}
$$

From Eq.(3.4) one may arrive at the following equation

$$
\begin{aligned}
& \lambda \ddot{E}+\dot{E}(t)=\left(1+\lambda \frac{\partial}{\partial t}\right)\left(\alpha_{1}(w, \phi)+\beta(w, \psi)\right)+ \\
& -\left(1+\lambda \frac{\partial}{\partial t}\right)\left[\Gamma\|\nabla \phi\|^{2}+\xi\|\nabla \psi\|^{2}+\eta(\nabla \phi, \nabla \psi)\right] .
\end{aligned}
$$

Thereby from the first of Eqs in (3.3), we may write

$$
\kappa\left(1+\lambda \frac{\partial}{\partial t}\right)\left(1+\lambda \frac{\partial}{\partial t}\right)(\phi, w)=\kappa\left(1+\lambda \frac{\partial}{\partial t}\right)\|w\|^{2} .
$$

Using Eq.(3.7), Eq.(3.6) may be rewritten as

$$
\begin{aligned}
& \lambda \ddot{E}+\dot{E}(t)=\left(1+\lambda \frac{\partial}{\partial t}\right)\left[\left(\alpha_{1}+\kappa\left(1+\lambda \frac{\partial}{\partial t}\right)\right)(w, \phi)+\beta(w, \psi)\right]+ \\
& -\left(1+\lambda \frac{\partial}{\partial t}\right)\left[\kappa\|\boldsymbol{u}\|^{2}+\Gamma\|\nabla \phi\|^{2}+\xi\|\nabla \psi\|^{2}+\eta(\nabla \phi, \nabla \psi)\right],
\end{aligned}
$$

and we may show that

$$
\lambda \ddot{E}+\dot{E}(t)=I-D \leq(m-1) D
$$

where the production term $I$ and the dissipation term $D$ are defined by

$$
\begin{aligned}
& I=\left(1+\lambda \frac{\partial}{\partial t}\right)\left[\left(\alpha_{1}+\kappa\left(1+\lambda \frac{\partial}{\partial t}\right)\right)(w, \phi)+\beta(w, \psi)\right], \\
& D=\left(1+\lambda \frac{\partial}{\partial t}\right)\left[\kappa\|\boldsymbol{u}\|^{2}+\Gamma\|\nabla \phi\|^{2}+\xi\|\nabla \psi\|^{2}+\eta(\nabla \phi, \nabla \psi)\right],
\end{aligned}
$$


and the number $m$ is defined by the relation

$$
m=\max _{H} \frac{I}{D}
$$

where $H$ is the space of the admissible fields. In order to ensure that the functional $D$ is positive definite, we require

$$
\begin{aligned}
& \frac{\mathrm{Le}^{2} \delta-1}{\mathrm{Le} \delta-1}>0, \quad \frac{\delta-1}{\mathrm{Le} \delta-1}>0, \\
& (\mathrm{Le}-1)^{2}\left(\mu_{l} \delta-1\right)^{2}-4 \mu_{1}\left(\operatorname{Le}^{2} \delta-1\right)(\delta-1)<0 .
\end{aligned}
$$

From Eq.(3.12), it follows that there exists a positive real number

$$
\alpha_{2}=\frac{4 \mu_{1}\left(\operatorname{Le}^{2} \delta-1\right)(\delta-1)-(\mathrm{Le}-1)^{2}\left(\mu_{l} \delta-1\right)^{2}}{4(\operatorname{Le} \delta-1)\left[\operatorname{Le}^{2} \delta-1+\mu_{1}(\delta-1)\right]}
$$

such that

$$
\alpha_{2}\left(\|\nabla \phi\|^{2}+\|\nabla \psi\|^{2}\right) \leq \frac{\mathrm{Le}^{2} \delta-1}{\mathrm{Le} \delta-1}\|\nabla \phi\|^{2}+\frac{(\mathrm{Le}-1)\left(\mu_{1} \delta-1\right)}{\mathrm{Le} \delta-1}(\nabla \phi, \nabla \psi)+\frac{\mu_{1}(\delta-1)}{\mathrm{Le} \delta-1}\|\nabla \psi\|^{2}
$$

If we assume $m<1$, from Eqs (3.9) and (3.13) there follows

$$
\begin{aligned}
& \lambda \ddot{E}+\dot{E}(t)=I-D \leq(m-1) \alpha_{2}\left(\|\nabla \phi\|^{2}+\|\nabla \psi\|^{2}\right), \\
& \leq \pi^{2} \tilde{\alpha}_{2}(m-1)\left(1+\lambda \frac{\partial}{\partial t}\right) E(t)
\end{aligned}
$$

where $\tilde{\alpha}_{2}=\tilde{\alpha}_{2} \min \left(\frac{1}{\mathrm{Le}}, \frac{1}{\mu_{1}}\right)$. From the above proceeding we deduce the following theorem:

Theorem 1: If $m<1$ the basic state is globally stable with decay at least like

$$
E(t) \leq E_{0} \exp \left(-\frac{t}{\lambda}\right)+E_{1} \exp \left(\pi^{2} \tilde{\alpha}_{2}(m-1) t\right)
$$

The condition $m<1$ is equivalent to $\Re<\Re_{E}=\operatorname{Le} \wp+\frac{4 \pi^{2}}{(\lambda \sigma+1)}$. 
In order to show that $\mathfrak{R}_{E}=\operatorname{Le} \wp+\frac{4 \pi^{2}}{(\lambda \sigma+1)}$, we observe that Eq.(3.11) is similar to that obtained in the fluid-dynamic case by Mulone and Rionero, (1998).The Euler-Lagrange equations for the maximization problem (3.11) are the following

$$
\begin{aligned}
& \left(1+\lambda \frac{\partial}{\partial t}\right)\left(-2 m \kappa \Delta w+\left(\alpha+\kappa\left(1+\lambda \frac{\partial}{\partial t}\right)\right) \Delta_{l} \phi+\beta \Delta_{l} \psi\right)=0 \\
& \left(1+\lambda \frac{\partial}{\partial t}\right)\left(-2 m \Gamma \Delta \phi-m \eta \Delta \psi-\left(\alpha+\kappa\left(1+\lambda \frac{\partial}{\partial t}\right)\right) w\right)=0 \\
& \left(1+\lambda \frac{\partial}{\partial t}\right)(-2 m \xi \Delta \psi-m \eta \Delta \phi-\beta w)=0
\end{aligned}
$$

where $\Delta_{1}$ is the two dimensional Laplacian.

Now, we eliminate $\phi$ and $\psi$ in Eq.(3.15) and follow the procedure of Mulone and Rionero (1998) to obtain

$$
m^{2}=\frac{\beta \eta \kappa+\beta \eta \alpha-2 \alpha \xi \kappa(1+\lambda \sigma)-\xi\left(\kappa^{2}+\alpha^{2}\right)-\kappa^{2} \xi \lambda \alpha(2+\lambda \sigma)-\beta^{2} \Gamma+\beta \lambda \kappa \sigma \eta}{4 \pi^{2} \kappa\left(\eta^{2}-4 \xi \Gamma\right)}
$$

where $\sigma$ is the frequency of oscillations. The determination of the optimal parameters $\kappa$ and $\mu_{l}$ and their substitution in Eq.(3.16) leads to

$$
m^{2}=\frac{(\Re-\wp)(1+\lambda \sigma)}{4 \pi^{2}} .
$$

Taking into account that $\wp$ is actually Le $\wp$, we get

$$
\mathfrak{R}<\mathfrak{R}_{E}=\operatorname{Le} \wp+\frac{4 \pi^{2}}{(\lambda \sigma+1)},
$$

whenever $m<1$. If $\lambda=0$, then Eq.(3.18) gives us the Newtonian liquid result of Lombardo et al. (2001).

Our next aim is to determine the nonlinear critical Rayleigh number, especially to obtain a region of coincidence of the linear and nonlinear critical stability parameters. To this end, we have to choose in an optimal way the Lyapunov parameters $\kappa, \mu_{1}$ and $\delta$. We may choose these parameters differently in various regions of the $\mathfrak{R}-\wp$ plane.

If we choose $\kappa=(\wp-\operatorname{Le} \Re) /(1+\lambda \sigma)$ and $\mu_{1}=(1 / \delta)$, then we get $\eta=0, \alpha_{1}+\kappa(1+\lambda \sigma)=0$ and from Eq.(3.16) we obtain the following expression for $m^{2}$

$$
m^{2}=\frac{(\Re-\delta \varsigma)^{2}(\text { Le } \delta-1)(1+\lambda \sigma)}{16 \pi^{2} \delta(\delta-1)(\widehat{\wp}-\text { Le } R)}
$$


From Eq.(3.19) it follows that $m^{2}<1$ whenever $\mathfrak{R}<\mathfrak{R}_{E}(\delta)$, where

$$
\mathfrak{R}_{E}=\delta\left[\oint-\frac{8 \pi^{2}(\delta-1) \mathrm{Le}}{(\operatorname{Le} \delta-1)(\lambda \sigma+1)}+\sqrt{\frac{64 \pi^{4}(\delta-1)^{2} \operatorname{Le}^{2}}{(\operatorname{Le} \delta-1)^{2}(\lambda \sigma+1)}+\frac{64 \pi^{2}(\delta-1) \hat{\gamma}}{(\operatorname{Le} \delta-1)(\lambda \sigma+1)}(1 / \delta-\mathrm{Le})}\right] .
$$

The positive definite conditions (3.12) require that $\delta$ does not exceed the value $1 / \mathrm{Le}^{2}$ and so we get nonlinear exponential stability whenever $\mathfrak{R}<\mathfrak{R}_{E(a \sin t)}$, where

$$
\mathfrak{R}_{E(a \sin t)}=\frac{1}{\mathrm{Le}} \wp-8 \pi^{2} \frac{\mathrm{Le}+1}{\mathrm{Le}^{2}}+\sqrt{64 \pi^{2} \frac{\mathrm{Le}+1}{\mathrm{Le}^{2}(\lambda \sigma+1)}\left[4 \pi^{2} \frac{\mathrm{Le}+1}{\mathrm{Le}^{2}}+\wp(1-1 / \mathrm{Le})\right]} .
$$

Equation (3.21) gives us the asymptotic nonlinear bound on $\mathfrak{R}$.

We now move order to make an unconditional stability analysis for all $\varepsilon$ and Le. So following the mentioned procedure, without change of dependent variables, on the linearized version of Eq.(2.4), we get

$$
\begin{aligned}
& \|w\|^{2}=\left(1+\lambda \frac{\partial}{\partial t}\right)(\Re \theta-\text { Lee } \wp \gamma, w), \\
& \frac{d}{d t} \frac{1}{2}\|\theta\|^{2}=(w, \theta)-\|\nabla \theta\|^{2}, \\
& \varepsilon \frac{d}{d t} \frac{1}{2}\|\gamma\|^{2}=\frac{1}{\mathrm{Le}}(w, \gamma)-\frac{1}{\mathrm{Le}}\|\nabla \gamma\|^{2} .
\end{aligned}
$$

For coupling parameters $\kappa>0, \mu_{1}>0$, as done earlier, we get the energy equation

$$
\frac{d}{d t} \frac{1}{2}\left(\kappa\|\theta\|^{2}+\varepsilon \mu_{1} \operatorname{Le}^{2} \wp\|\gamma\|^{2}\right)=I-D
$$

where

$$
\begin{aligned}
& I=\left(\mathfrak{R}\left(1+\lambda \frac{\partial}{\partial t}\right)+\kappa\right)(w, \theta)+\operatorname{Le} \wp\left(\mu_{1}-\left(1+\lambda \frac{\partial}{\partial t}\right)\right)(w, \gamma), \\
& D=\left[\|\boldsymbol{u}\|^{2}+\kappa\|\nabla \theta\|^{2}+\operatorname{Le} \wp \mu_{l}\|\nabla \gamma\|^{2}\right] .
\end{aligned}
$$

If we now define

$$
\max _{H} \frac{I}{D}=\frac{1}{\Lambda},
$$

then with 


$$
E(t)=\frac{1}{2}\left(\kappa\|\theta\|^{2}+\varepsilon \mu_{1} \operatorname{Le}^{2} \wp\|\gamma\|^{2}\right)
$$

we find from Eq.(3.25) that

$$
\frac{d E}{d t} \leq D\left(\frac{1}{\Lambda}-1\right)
$$

For $\frac{1}{\Lambda}<1$, Eq.(3.28) produces unconditional stability. The Euler equations for the maximization problem (3.26) are

$$
\begin{aligned}
& \left(\Re\left(1+\lambda \frac{\partial}{\partial t}\right)+\kappa\right) \theta \boldsymbol{k}+\operatorname{Le} \wp\left(\mu_{1}-\left(1+\lambda \frac{\partial}{\partial t}\right)\right) \gamma \boldsymbol{k}-2 \boldsymbol{u}=\nabla p, \\
& \left(\Re\left(1+\lambda \frac{\partial}{\partial t}\right)+\kappa\right) w+2 \kappa \Delta \theta=0, \\
& \left(\mu_{1}-\left(1+\lambda \frac{\partial}{\partial t}\right)\right) w+2 \mu_{1} \Delta \gamma=0 .
\end{aligned}
$$

From Eqs (3.29)-(3.31) following standard procedure we get the following equation for $\Re$

$$
\frac{(\Re(1+\lambda \sigma)+\kappa)^{2}}{\kappa}+\frac{\operatorname{Le} \wp\left(\mu_{1}-(1+\lambda \sigma)\right)}{\mu_{1}}=\frac{4\left(n^{2} \pi^{2}+a^{2}\right)^{2}}{a^{2}} .
$$

For $\mu_{1}=(1+\lambda \sigma)$, we get $\Re$ largest. Minimizing LHS with respect to $n^{2}$ and $a^{2}$ we get

$$
\Re=\frac{4 \pi \sqrt{\kappa}-\kappa}{1+\lambda \sigma} .
$$

The optimal value of $\mathfrak{R}$ with respect to $\kappa$ is

$$
\Re=\frac{4 \pi^{2}}{1+\lambda \sigma} .
$$

What we have shown is that when $R<\frac{4 \pi^{2}}{1+\lambda \sigma}$, then the solution basic state is unconditionally stable regardless of what value $\wp(>0)$ has, and for all values of $\varepsilon$ and Le. Further, for Le $\delta<<1$ (dilute solutions). Equations (3.18) and (3.34) reveal the coincidence of the linear and non-linear critical stability parameters. Another important result emanating from the study is showing the possibility of unconditional non-linear exponential stability, using energy stability analysis, in a problem that lacks symmetry. 
It is important to note here that the results of Lombardo et al. (2001) and Wang and Tan (2008) are recovered as limiting case of the study. It would, however, be more interesting to consider the Jeffrey model in the problem. This is under progress.

\section{Nomenclature}

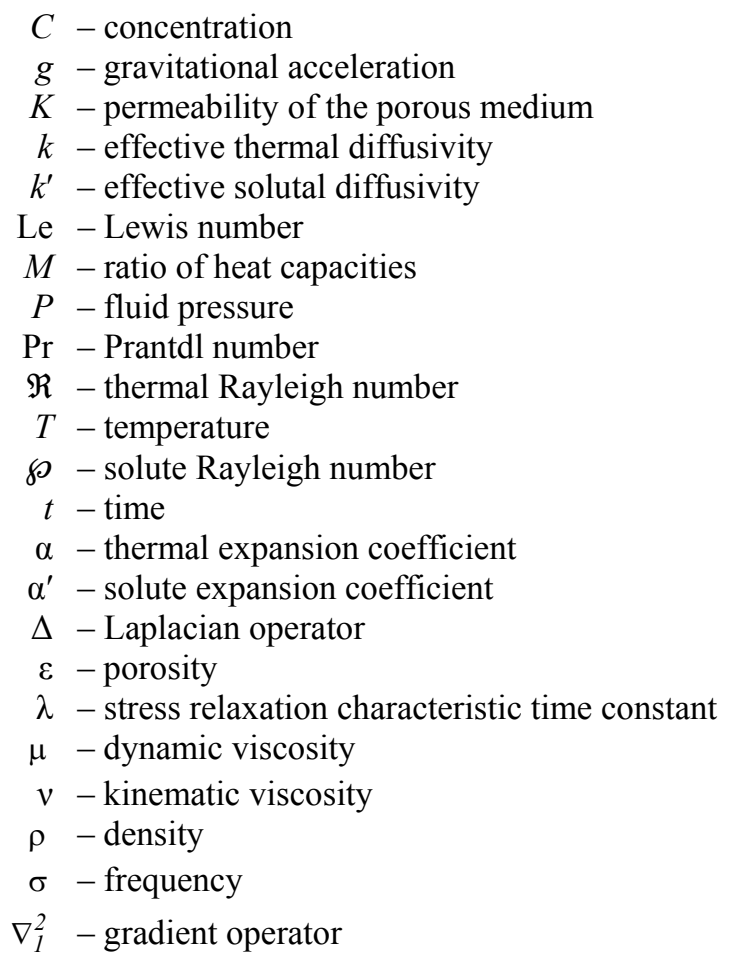

\section{References}

Alloui Z., Vasseur P., Robillard L. and Bahloul A. (2010): Onset of double-diffusive convection in a horizontal Brinkman cavity. - Chemical Engineering Communications, vol.197, pp.387-399.

Awad F.G., Sibanda P. and Motsa S.S. (2010): On the linear stability analysis of a Maxwell fluid with double-diffusive convection. - Applied Mathematical Modelling, vol.34, pp.3509-3517.

Bejan A. (2004): Convection Heat Transfer. - New Jersey: John Wiley and Sons.

Fu C., Zhang Z. and Tan W. (2007): Numerical simulation of thermal convection of a viscoelastic fluid in a porous square box heated from below. - Physics of Fluids, vol.19, 104-107.

Haro M.L., Rio J.A.P. and Whitaker S. (1996): Flow of Maxwell fluid in porous media. - Transport in Porous Media 25, pp.167-192.

Kim M.S., Lee S.B., Kim S. and Chung B.J. (2003): Thermal instability of viscoelastic fluids in porous media. - Int. J. Heat and Mass Transfer, vol.46, pp.5065-5072.

Kumar P. and Singh M. (2006): On a viscoelastic fluid heated from below in a porous medium. - Journal of NonEquilibrium Thermodynamics, vol.31, No.2, pp.189-203.

Lombardo S., Mulone G. and Straughan B. (2001): Non-linear stability in the Benard problem for double-diffusive mixture in a porous medium. - Mathematical Methods in the Applied Sciences, Math. Meth. Appl. Sci., vol.24, pp.1229-1246.

Long J.S., Chen J.H., Chen H.K., Tam L.M. and Chao Y.C. (2009): A unified system describing dynamics of chaotic convection. - Chaos, Solitons and Fractals, vol.41, pp.123-130. 
Long J.S., Tam L.M., Chen J.H., Chen H.K., Lin K.T. and Yuan Kang (2008): Chaotic convection of viscoelastic fluids in porous media. - Chaos, Solitons and Fractals, vol.37, pp.113-124.

Malashetty M.S. and Kulkarni S. (2009): The convective instability of Maxwell fluid-saturated porous layer using a thermal non-equilibrium model. - J. Non-Newtonian Fluid Mech., vol.162, pp.29-37.

Malashetty M.S., Swamy M. and Heera R. (2009): The onset of convection in a binary viscoelastic fluid saturated porous layer. - ZAMM. Z. Angew. Math. Mech., vol.89, No.5, pp.356-369.

Malashetty M.S., Tan W. and Swamy M. (2009): The onset of double diffusive convection in a binary viscoelastic fluid saturated anisotropic porous layer. - Physics of Fluids, vol.21, pp.084101.

Mulone G. and Rionero S. (1998): Unconditional nonlinear exponential stability in the Benard problem for a mixture: necessary and sufficient conditions. - Rendiconti Mat. Acc. Lincei, series 9, 9, pp.221-236.

Nield D.A. and Bejan A. (2006): Convection in Porous Media. - New York: Springer-Verlag.

Sharma R.C. and Sunil (1994): Thaermal instability of Oldroydian viscoelastic fluid with suspended particles in hydromagnetics in porous medium. - Polymer-Plastics Technology and Engineering, vol.33, No.3.

Shivakumara I.S. and Sureshkumar S. (2008): Effect of throughflow and quadratic drag on the stability of a doubly diffusive Oldroyd-B fluid-saturated porous layer. - J. Geophys. Eng., vol.5, pp.268-280.

Siddheshwar P.G. and Sri Krishna C.V. (2001): Rayleigh -Benard convection in a viscoelastic fluid-filled high-porosity medium with non uniform basic temperature gradient. - IJMMS, vol.25, No.9, pp.609-619.

Siddheshwar P.G. and Sri Krishna C.V. (2003): Linear and non - linear analyses of convection in a micropolar fluid occupying a porous medium. - Int. J. Nonlinear Mech., vol.38, pp.1561-1579.

Sri Krishna C.V. (2001): Effects of non-inertial acceleration on the onset of convection in a second-order fluidsaturated porous medium. - International Journal of Engineering Science, vol.39, pp.599-609.

Tan W.C. and Masouka T. (2007): Stability analysis of Maxwell fluid in a porous medium heated from below. - Phys. Lett. A 360, pp.454-460.

Vafai K. (Ed.) (2000): Handbook of Porous Media. - New York: Marcel Dekker.

Wang S. and Tan W.C. (2008): Stability analysis of double-diffusive convection of Maxwell fluid in a porous medium heated from below. - Phys Lett. A 372, pp.3046-3050.

Wang S. and Tan W. (2011): Stability analysis of Soret-driven double-diffusive convection of Maxwell fluid in a porous medium. - International Journal of Heat and Fluid Flow, vol.32, pp.88-94.

Received: September 28, 2012

Revised: October 2, 2012 